\title{
Center Black Hole Mass Determinations for Fermi Blazars*
}

\author{
J. H. Fan ${ }^{1,2}$, Y. Liu ${ }^{1,2}$, J. H. Yang ${ }^{3}$, Y. H. Yuan ${ }^{1,2}$, Y. Li ${ }^{4}$ and, \\ Q. F. Zhang ${ }^{4}$ \\ ${ }^{1}$ Center for Astrophysics, Guangzhou University, Guangzhou 510006, China \\ email: jhfan_cn@yahoo.com.cn \\ ${ }^{2}$ Astronomy Science and Technology Research Laboratory of Department of Education of \\ Guangdong Province, Guangzhou 510006, China \\ ${ }^{3}$ Department of Physics and Electronics Science, Hunan University of Arts and Science, \\ Changde 415000, China \\ ${ }^{4}$ Department of Computer Science, Jinan University, Guangzhou 510632, China
}

\begin{abstract}
In this paper, we estimated the black hole mass for some blazars with available variability time scales by assuming that the $\gamma$-ray emissions are from a distance of $200 \mathrm{R}_{g}$ from the center. The results show that the central black hole masses are in the range of $\log \left(M / M_{\odot}\right)$ $=6.45$ to 8.30 .
\end{abstract}

Keywords. Galaxies:active-galaxies:BL Lac objects-galaxies:quasars-Fermi(LAT)

\section{Introduction}

Blazars are extreme subclass of active galactic nuclei (AGNs) showing special observation properties. The power for all types of AGNs is almost universally ascribed to accretion onto supermassive black holes with masses $M \sim 10^{6-9} M_{\odot}$ (Dermer \& Gehrels, 1995). Blazars show variability in the $\gamma$-ray band on timescales from hours to months(Mukherjee, et al. 1997). Some authors claimed that the $\gamma$-rays are produced at a region of $\sim 100 \mathrm{R}_{g}\left(\mathrm{R}_{g}=G M / c^{2}\right)$ (Hartman 1996) and a few $100 \mathrm{R}_{g}$ ( Ghisellini \& Gabriele, 1996) from the central black hole, it is also found to be $205 \mathrm{R}_{g}$ (Xie et al. 1996). The distance is useful for the determination of the central black hole mass. In this paper, we will use the Fermi observations data (Nolan et al. 2012) and the assumption of the $\gamma$-ray emission region, $R_{\gamma} \sim 200 R_{g}$ to estimate the central black hole mass. Here, we adopt $\mathrm{H}_{0}=73 \mathrm{~km} \mathrm{~s}^{-1} \mathrm{Mpc}^{-1}$.

\section{Method and Results}

We now describe the method to estimate the central black hole mass for a blazar with variability time scale (Fan et al. 1999). If we take the variability timescale as the measurements of the emission size, $R$, then $R$ obeys to the inequality, $R \leqslant \frac{c \Delta T \delta}{1+z}$, here $\mathrm{c}$ is the speed of light, $\delta$ is the Doppler factor, z the redshift, and $\Delta T$ the time scale. If $R=$ $200 R_{g}$, then we have $\left(\mathrm{M} / \mathrm{M}_{\odot}\right) \leqslant 3.6 \cdot 10^{6}\left(\frac{\Delta \mathrm{T}}{1 \mathrm{hr}}\right) \frac{\delta}{1+\mathrm{z}}$. In a beaming frame, we have that the observed luminosity, $\mathrm{L}^{\mathrm{ob}}$, is associated with the intrinsic luminosity, $\mathrm{L}^{\mathrm{in}}, \mathrm{L}^{\mathrm{ob}}=\delta^{4+\alpha} \mathrm{L}^{\mathrm{in}}$ (here we take $L^{i n}=\lambda L_{E d d}=1.26 \cdot 10^{45} \lambda M_{7}$ ), then we have, $\delta \geqslant\left[\frac{L_{45}^{o b}(1+z)}{0.454\left(\frac{\Delta T}{1 h r}\right) \lambda}\right]^{\frac{1}{5+\alpha}}$,

${ }^{*}$ The work is partially supported by the National Natural Science Foundation of China (NSFC 11173009), the Bureau of Education of Guangzhou Municipality (No.11 Sui-Jiao-Ke[2009]), Guangdong Province Universities and Colleges Pearl River Scholar Funded Scheme (GDUPS)(2009), and Yangcheng Scholar Funded Scheme (10A027S). 
Table 1. Black Hole Masses for Fermi Blazars

\begin{tabular}{lccccccc}
\hline Name & Class & $\mathrm{z}$ & $\mathrm{F}(1-100 \mathrm{GeV})$ & $\alpha_{\gamma}$ & $\Delta T$ & $\delta_{\gamma}$ & $\log \left(M / M_{\odot}\right)$ \\
\hline 1 & 2 & 3 & 4 & 5 & 6 & 7 & 8 \\
\hline $0208-512$ & FSRQ & 1.003 & 37.9 & 2.4 & 134 & 1.56 & 8.27 \\
$0219+428$ & BL & 0.444 & 256 & 1.85 & 30 & 2.25 & 7.93 \\
$0235+164$ & BL & 0.94 & 187 & 2.02 & 72 & 2.42 & 8.21 \\
$0528+134$ & FSRQ & 2.06 & 25.7 & 2.22 & 24 & 2.91 & 7.61 \\
$0537-441$ & BL & 0.894 & 371 & 2.01 & 16 & 3.41 & 7.71 \\
$0650+453$ & FSRQ & 0.933 & 34.5 & 2.28 & 66 & 1.70 & 8.02 \\
$0735+178$ & BL & 0.424 & 51.6 & 2.05 & 24 & 1.59 & 7.68 \\
$0851+202$ & BL & 0.306 & 35.5 & 2.23 & 1 & 2.03 & 6.45 \\
$1101+384$ & BL & 0.031 & 297 & 1.77 & 2 & 1.35 & 6.67 \\
B1127-145 & FSRQ & 1.187 & 18 & 2.7 & 6 & 2.34 & 7.06 \\
$1156+295$ & FSRQ & 0.729 & 60.4 & 2.29 & 11 & 2.19 & 7.40 \\
$1219+285$ & BL & 0.102 & 55.4 & 2.02 & 3.6 & 1.25 & 6.87 \\
$1226+023$ & FSRQ & 0.158 & 151 & 2.45 & 24 & 1.12 & 7.62 \\
$1253-055$ & FSRQ & 0.537 & 256 & 2.22 & 12 & 2.41 & 7.53 \\
$1502+106$ & FSRQ & 1.83 & 401 & 2.15 & 12 & 4.89 & 7.57 \\
$1510-089$ & FSRQ & 0.361 & 406 & 2.29 & 8 & 2.26 & 7.38 \\
$1520+31$ & FSRQ & 1.487 & 176 & 2.25 & 6.7 & 4.06 & 7.29 \\
$1633+382$ & FSRQ & 1.814 & 116 & 2.25 & 16 & 3.67 & 7.57 \\
$1652+398$ & BL & 0.033 & 87.7 & 1.74 & 6 & 0.94 & 6.99 \\
$1700+6830$ & FSRQ & 0.301 & 38 & 2.4 & 7.4 & 1.42 & 7.16 \\
$2022-070$ & FSRQ & 1.388 & 101 & 2.15 & 113 & 2.35 & 8.30 \\
B2155-304 & BL & 0.117 & 235 & 1.84 & 3.3 & 1.87 & 7.00 \\
$2200+420$ & BL & 0.07 & 105 & 2.11 & 3.2 & 1.19 & 6.81 \\
$2230+114$ & FSRQ & 1.04 & 28.8 & 2.33 & 48 & 1.81 & 7.89 \\
$2251+158$ & FSRQ & 0.859 & 965 & 2.23 & 1.9 & 4.99 & 6.96 \\
\hline
\end{tabular}

$L_{45}^{o b}(\mathrm{erg} / \mathrm{s})=\mathrm{L}^{\mathrm{ob}} / 10^{45}$. From the available data, we can get the results for the central black hole masses and the Doppler factors, and listed them in Table 1. In Table 1, column (1) gives the name of the source, column (2) classification (BL, FSRQ-flat spectrum radio quasars), column (3) the redshift, column (4) and (5) the $\gamma$-ray photons in $1-100 \mathrm{GeV}$ in units of $10^{-10}$ photon $/ \mathrm{cm}^{2} / \mathrm{s}$ and photon spectrum index from Ackermann, et al., (2011) and Nolan, et al. (2012), column (6) the time scale $\Delta T$ in units of hours (Yang \& Fan 2010), column (7) the $\gamma$-ray Doppler factor, column (8) the central black hole mass, log $\left(M / M_{\odot}\right)$.

Discussions and Conclusion From Table 1, we can see that the central black hole masses have a range of $\log \left(M / M_{\odot}\right)=6.45$ to $8.30 .<\log \left(M / M_{\odot}\right)>=7.23 \pm 0.60$ for BLs, and $<\log \left(M / M_{\odot}\right)>=7.58 \pm 0.40$ for FSRQs. For the Doppler factors, $\left\langle\delta_{\gamma}\right\rangle=1.83 \pm 0.74$ for BLs and $\left\langle\delta_{\gamma}\right\rangle=2.65 \pm 1.22$ for FSRQs. The results are similar to those in our previous work (Yang \& Fan 2010). There is no clear difference in masses or Doppler factors between BLs and FSRQs. Does that mean that the central black hole mass is not an important factor in the evolution process if there is really an evolution between FSRQs and BLs?

\section{References}

Ackermann, M., Ajello, M., Allafort, A., et al. 2011, ApJ, 743, 171

Dermer, C. D. \& Gehrels, N. 1995, ApJ, 447, 103

Fan, J. H., Xie, G. Z., \& Bacon, R. 1999, AछAS, 136, 13

Ghisellini, G. \& Madau, P. 1996, MNRAS, 280, 67

Hartman, R. C. 1996, ASPC 110, 333

Mukherjee, R., Bertsch, D. L., Bloom, S, D., et al. 1997, ApJ, 490, 116

Nolan, P. L., Abdo, A. A., Ackermann, M., et al. 2012, ApJS, 199, 31

Xie, G, Z, Bai, J. M., Zhang, X., et al. 1998, A\&A, 334, 29

Yang, J. H. \& Fan, J. H. 2010, Sci. Chin. Phys. Mech. \& Astron. 53, 1921 